Sādhanā Vol. 40, Part 3, May 2015, pp. 891-909. (C) Indian Academy of Sciences

\title{
Flow and oscillations in collapsible tubes: Physiological applications and low-dimensional models
}

\author{
T J PEDLEY ${ }^{1, *}$ and D PIHLER-PUZOVIĆ ${ }^{2}$
}

${ }^{1}$ Department of Applied Mathematics and Theoretical Physics, University of Cambridge, Centre for Mathematical Sciences, Wilberforce Road, Cambridge CB3 0WA, UK

${ }^{2}$ Manchester Centre for Nonlinear Dynamics and School of Physics and Astronomy, University of Manchester, Oxford Road, Manchester M13 9PL, UK

e-mail: t.j.pedley@damtp.cam.ac.uk; draga.pihler-puzovic@manchester.ac.uk

MS received 25 July 2014; revised 12 January 2015; accepted 6 February 2015

\begin{abstract}
The motivation for this subject comes from physiology: Air-flow in the lungs, where flow limitation during forced expiration is a consequence of large-airway collapse, and wheezing, which is a manifestation of self-excited mechanical oscillations; Blood flow in veins, such as those of giraffes, in which the return of blood to the heart from the head must be accompanied by partial venous collapse, and in arteries, which exhibit self-excited oscillations (Korotkov sounds) when compressed by a blood-pressure cuff. Laboratory experiments are frequently conducted in a Starling resistor, a finite length of flexible tube, mounted between two rigid tubes and contained in a pressurised chamber. Steady conditions upstream and downstream give rise not only to steady flows, but also to a rich variety of self-excited oscillations, which theoreticians have been seeking to understand for at least five decades. Some of the observations have been reproduced in full Navier-Stokes computations for a two-dimensional model, but these do not provide physical understanding. We seek a self-consistent mathematical model for the oscillations. We concentrate first on 1D models, in which the key dependent variables are the cross-sectional area $A$ and the cross-sectionally averaged velocity $u$ and pressure $p$, all taken to be functions of longitudinal coordinate $x$ and time $t$. The governing equations are those of conservation of mass and momentum and a tube law representing the elastic properties of the vessel. In the momentum equation, the viscous resistance term is conventionally modelled either as a linear function of fluid velocity, accurate at low Reynolds number, or with an ad hoc representation of the energy loss at flow separation. Even with such crude approximations, the predictions of 1D models agree quite well both with observations in the giraffe and with some of the 2D computations and 3D experiments. For a more rational model, we examine a 2D model problem, in which part of one wall of a parallel sided channel is replaced by a membrane under tension. One
\end{abstract}

*For correspondence 
approach, for large Reynolds-number flow, and a long membrane, is to consider small deflections of the membrane and use interactive boundary-layer theory. This leads to interesting predictions, such as the impossibility of simultaneously prescribing the flow rate and the upstream pressure, but not to oscillations, except in cases where wall inertia is important (flutter). Another approach is to assume a parabolic velocity profile everywhere, leading to a rational choice for the inertia and viscous terms in the 1D momentum equation. If, further, the undisturbed membrane is taken to be flat, by a suitable choice of external pressure distribution, the system leads to an oscillatory instability even without wall inertia. Whether these oscillations have the same physics as those computed numerically at lower Reynolds number remains to be seen.

Keywords. Collapsible tubes; flow-structure interactions; 1D models; 2D models.

\section{Introduction}

Whenever the pressure internal to a flexible tube falls below the external pressure, the tube crosssection tends to buckle and change shape, i.e. to collapse. (Here the word "collapse" signifies a dramatic reduction in cross-sectional area, not normally leading to total occlusion.) A typical relation between cross-sectional area and transmural pressure is shown in figure 1: for transmural pressures close to zero the tube is extremely compliant - so much so that the dynamic pressure changes associated with fluid flow in the tube may be enough to generate large area changes.

Collapsible tubes have been studied by mechanical scientists for the last 50 years, the motivation generally being physiological. Large airways in the lung collapse during forced expiration, because the external pressure is essentially the same as the pleural pressure which, applied to the exterior of the alveolae, is responsible for driving the flow, and the internal pressure is lower than that as a result of the viscous resistance to flow. This collapse is responsible for the phenomenon of flow-limitation, whereby an increase in expiratory effort above a critical level does not result in increased flow rate; indeed, there are examples of "negative effort-dependence" in which the flow rate actually falls when the effort is increased, depending on lung volume (see figure 2, from Hyatt et al (1958)). In addition, wheezing during expiratory flow, especially in subjects with some lung disease, is a manifestation of self-excited oscillations which frequently arise during flow through flexible tubes as discussed below (Gavriely et al 1989).

In the cardiovascular system, arteries are well pressurised, so they do not normally collapse, except when they are actively squeezed, for example under a blood-pressure cuff. The Korotkov sounds listened for by the physician are also manifestations of flow-induced oscillations. Veins, however, are thin-walled tubes with mean internal pressure that is only just above atmospheric at the level of the heart, and it is therefore expected to be subatmospheric above that level, as a result of the gravitational fall in hydrostatic pressure with height in a fluid. This has significant implications for blood flow in the neck, especially for long-necked individuals. Figure 3 shows measurements of pressure (above atmospheric) in the carotid artery and jugular vein of adult giraffes (Hargens et al 1987): indeed pressure falls with height in the artery, but it does not fall in the vein, even increasing a little. This requires explanation. So too do the facts that, in the giraffe, central arterial blood pressure is about 2.5 times that in humans and almost all other mammals, and that the heart may constitute over 2 percent of body mass, compared with about 0.5 percent in humans. 


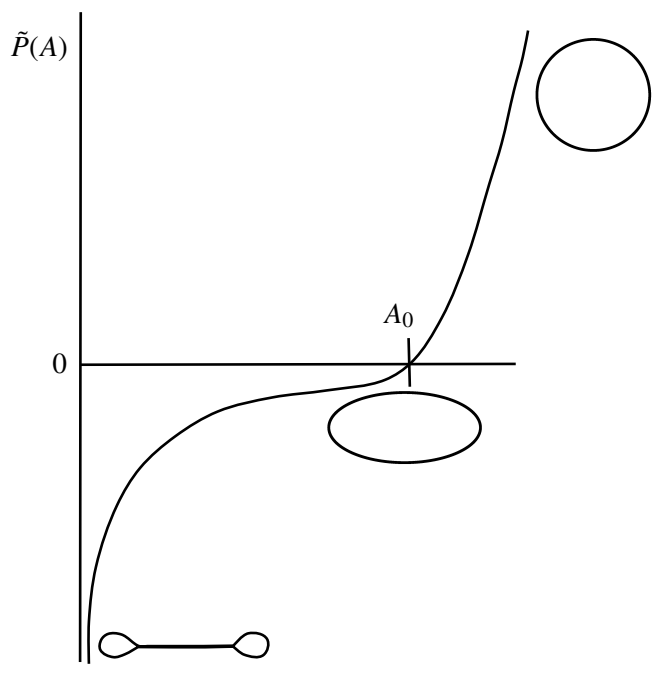

Figure 1. Sketch of the 'tube law' for an elastic tube, relating transmural pressure $\tilde{P}$ and cross-sectional area $A$. Sketches of cross-sectional shape are given for three regions of the curve. From Pedley (2000), figure 4.

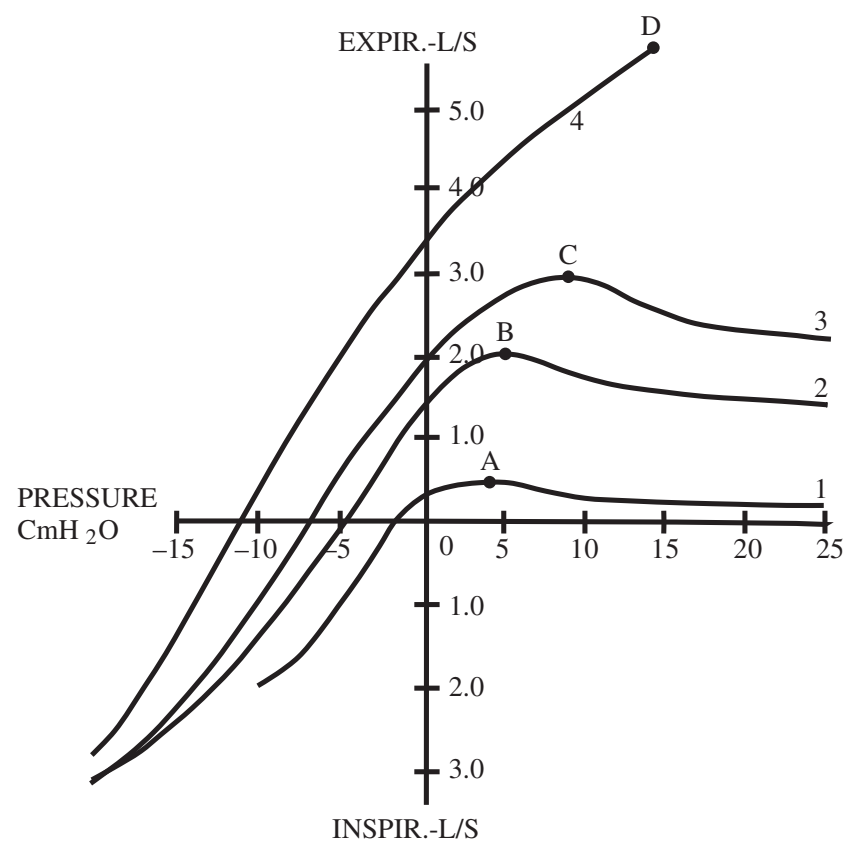

Figure 2. Relationship of transpulmonary (driving) pressure and flow rate for a normal subject at different degrees of lung inflation. Curves 1, 2, 3 and 4 were measured at 0.6, 1.2, 1.7 and 2.8 L, respectively, above the maximum expiratory point. From Hyatt et al (1958), figure 1. 


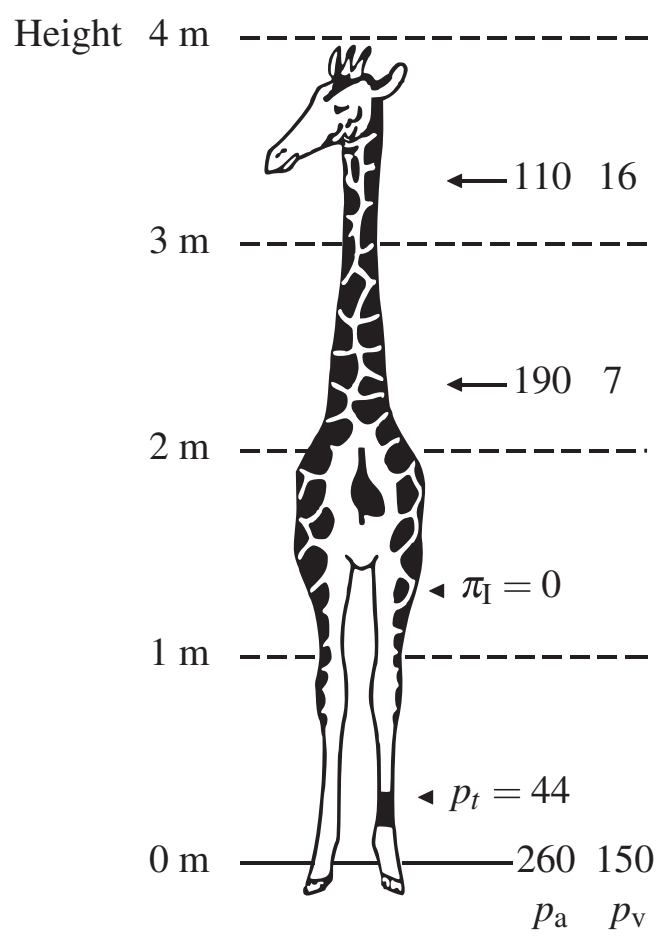

Figure 3. Mean arterial, $p_{a}$, and venous, $p_{v}$, pressure in an upright adult giraffe at hydrostatic levels between the head and feet, measured in $\mathrm{mm} \mathrm{Hg}$. $p_{a}$ was averaged over 8 animals, $p_{v}$ over 3 . Adapted from Hargens et al (1987), figure 1.

As a very simple model, consider a single, uniform pipe containing viscous fluid flowing steadily at volume flow rate $Q$. Let the pressure at the upstream end be $P_{1}$ and that at the downstream end, a distance $L$ along the pipe and a vertical distance $h$ above, be $P_{2}$. Then, ignoring inertia, we have

$$
P_{1}-\left(P_{2}+\rho g h\right)=R L Q
$$

where $\rho$ is the fluid density, $g$ is the gravitational acceleration and $R$ is the viscous resistance of the pipe per unit length, which will in general be a function of the tube cross-sectional area $A$. Now consider the system sketched in figure 4, which can be both a laboratory experiment or a model of the circulatory system in the giraffe head and neck (Pedley et al 1996). There is a pump (representing the left ventricle) at point 1 (bottom left), generating pressure $P_{1}$. From here the tube goes upwards (the carotid arteries) before looping round to point 2 at height $h$ (where the jugular veins exit the skull), after a distance $L$, and then descending to a reservoir (the right atrium) at the same level as point 1 , at point 3 . If the tube system is rigid, the only difference between $P_{1}$ and $P_{3}$ is $R(L+h) Q$, where $R$ is suitably averaged along the system, and is positive. Thus all that is required to pump fluid along at some positive flow rate $Q$ is that $P_{1}$ should exceed $P_{3}$ : the system acts like a siphon and the pressure in the 'head' and downflow tube would be subatmospheric. Moreover, whatever the nature of the tube downstream of point 2, and still neglecting unsteadiness and inertia, Eq. (1) will hold. If there were no tube beyond point 2, $P_{2}$ would be atmospheric, i.e. zero, so in order for there to be a non-zero $Q, P_{1}$ would have to exceed $P_{2}$ by at least $g \rho h$; in other words the fluid would have to be pumped uphill, $Q$ 


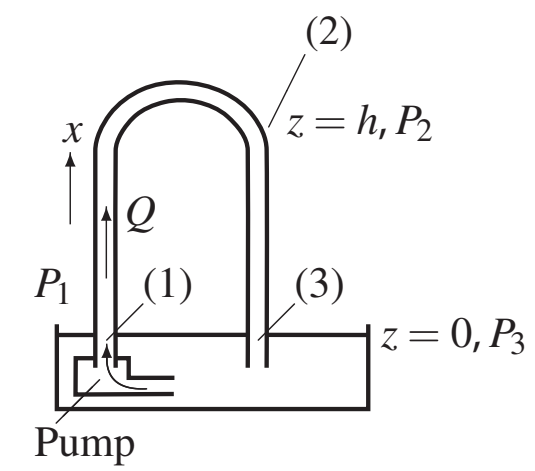

(a)

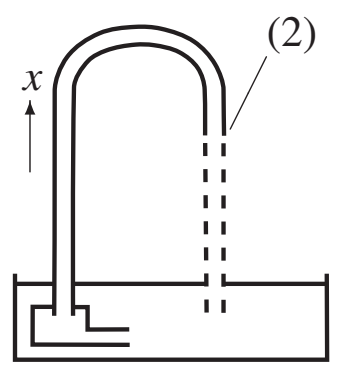

(b)

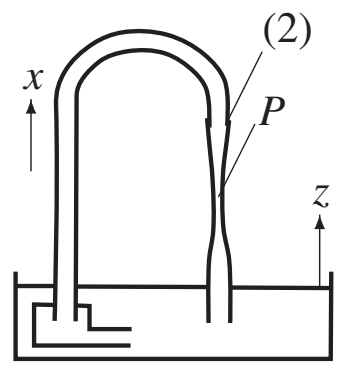

(c)

Figure 4. Sketch of possible experiments with an inverted U-tube, supplied with flow rate $Q$ by a pump at $z=0$ generating pressure $P_{1}$. The point (2) is at height $z=h$ and the pressure there is $P_{2}$; the distance along the tube from the pump to point (2) is $L$. The pumped fluid is collected in a reservoir at level $z=0$, where pressure is atmospheric, $P_{3}$. (a) Complete rigid tube; $P_{2}$ is subatmospheric. (b) Tube is cut off at (2); $P_{2}$ is atmospheric. (c) Tube replaced by collapsible drain tubing below (2); $P_{2}$ is atmospheric. From Pedley et al (1996), figure 2.

being given by (1). Finally if, downstream of point 2, there were a highly compliant tube, the pressure in it could not be subatmospheric since it would already have collapsed. In fact, the pressure would be precisely atmospheric all along it (Hicks \& Badeer 1989). One might ask how could that be, given the relevant equation corresponding to (1)? The answer is that in such a case (1) determines the value of $R$, say $R_{c}$, in the collapsible tube:

$$
R_{c}=\rho g / Q \text {. }
$$

Since viscous resistance is inversely related to cross-sectional area, Eq. (2) will determine $A$.

That model, applied to the giraffe, suggests that (a) the jugular vein is normally collapsed, (b) the pressure in it is approximately atmospheric, (c) the system is not a siphon, and (d) the heart has to pump the blood uphill. Since the hill is very high, the pressure generated by the left ventricle $\left(P_{1}\right)$ must be correspondingly high (as observed). Moreover, since the (average) work done per unit time by the heart in sending flow through the head is $P_{1} Q$, the heart has to work much harder and therefore requires much more muscle per unit mass than in other mammals. The only one of the questions raised above that has not been answered is why the venous pressure apparently rises with height in the giraffe jugular vein. Pedley et al (1996) attributed this to longitudinal variations in the cross section and elastic properties of the vein, but this has not been verified experimentally. One might also ask about the effect of inertia, especially in timedependent manoeuvres. This discussion requires a less idealised one-dimensional model of flow in collapsible tubes, as is outlined in section 2 below. The application to the giraffe was made by Brook et al (1999) and will not be discussed further here.

Laboratory experiments to investigate pressure-flow relations and self-excited oscillations in collapsible tubes have commonly been performed in a Starling resistor, a finite length of flexible (e.g. rubber) tube, mounted between two rigid tubes and contained in a pressurised chamber (figure 5). The pressure is typically held fixed far downstream, and either the flow rate or the pressure may be fixed upstream. In either case, for a wide range of governing parameter values (such as Reynolds number $R e$ ), the system exhibits a rich variety of self-excited oscillations 


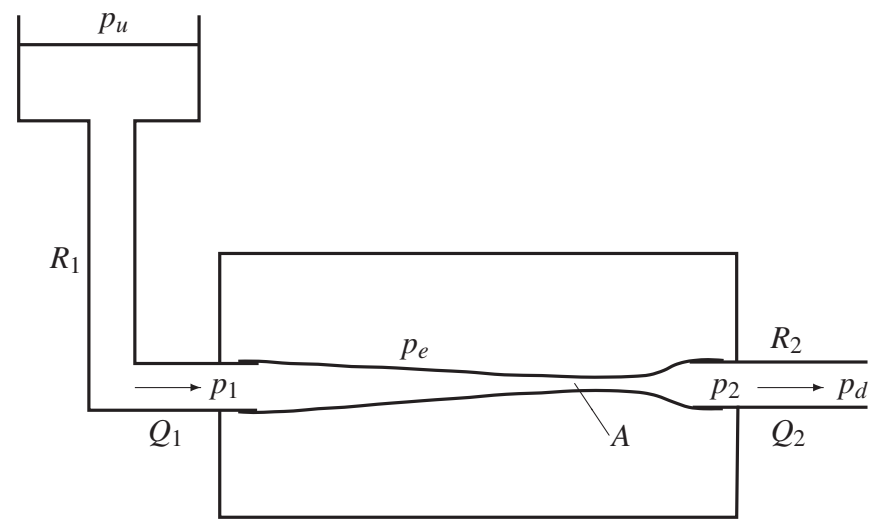

Figure 5. Sketch of a standard laboratory experiment. $p_{1}, Q_{1}$ are pressure and flow rate upstream of the collapsible segment; $p_{2}, Q_{2}$ are pressure and flow rate downstream; $p_{u}, p_{d}$ are total pressure far upstream and downstream; $p_{e}$ is pressure in the chamber surrounding the collapsible segment. $R_{1}$ and $R_{2}$ represent the rigid segments up- and downstream, whose resistance can be prescribed. From Pedley (2000), figure 7.

(figure 6, from Bertram et al (1991)). The object of theoretical research is to understand both the steady flows and the mechanisms underlying the oscillations. Direct numerical simulations of the full, three-dimensional (3D), flow-structure system would still be computationally very time-consuming and expensive, so for over 20 years considerable attention has been paid to a 2D idealisation of the Starling resistor (figure 7). This system consists of a parallel-sided rigid
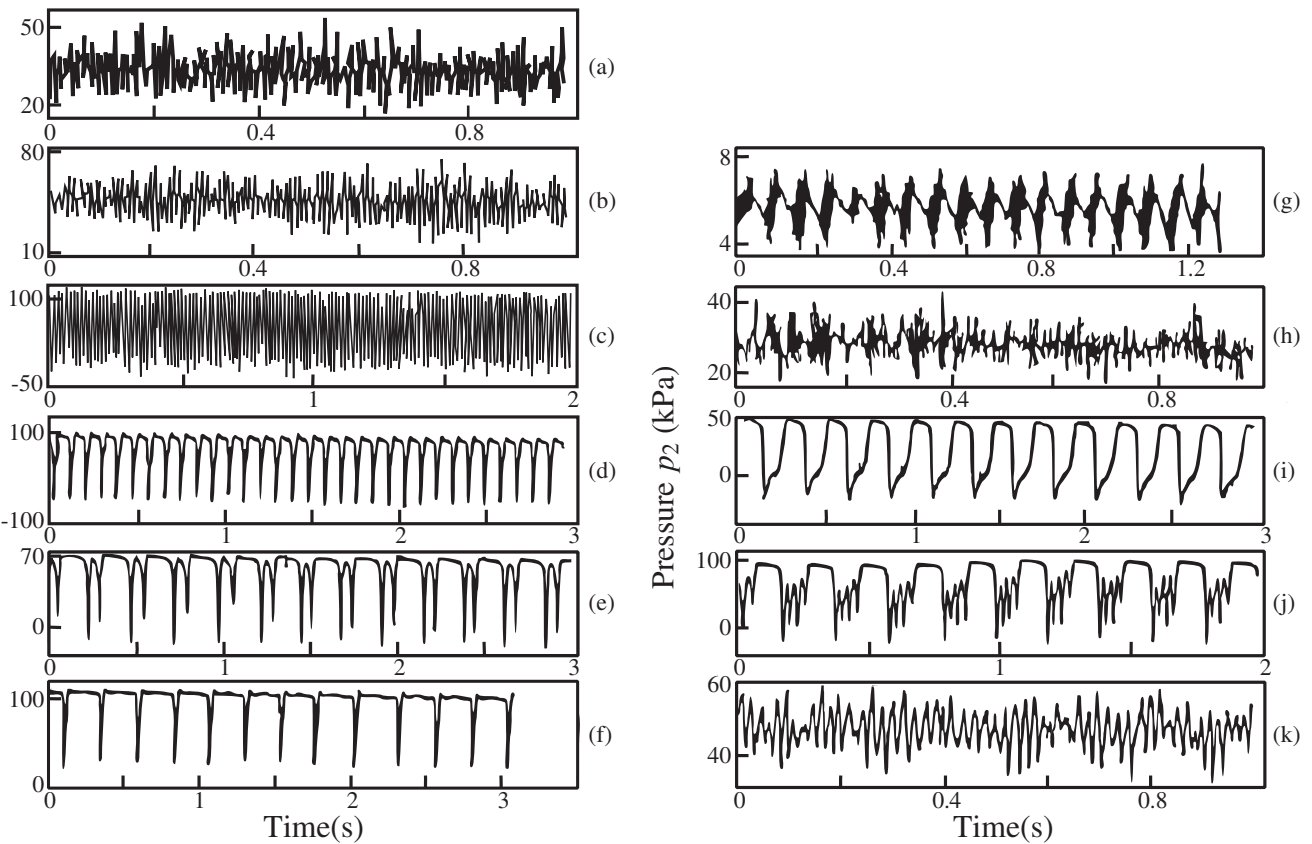

Figure 6. Pressure, $p_{2}$, at the downstream end of the collapsible segment, plotted against time $t$ during self-excited oscillations for various values of the governing parameters. From Bertram et al (1991). 


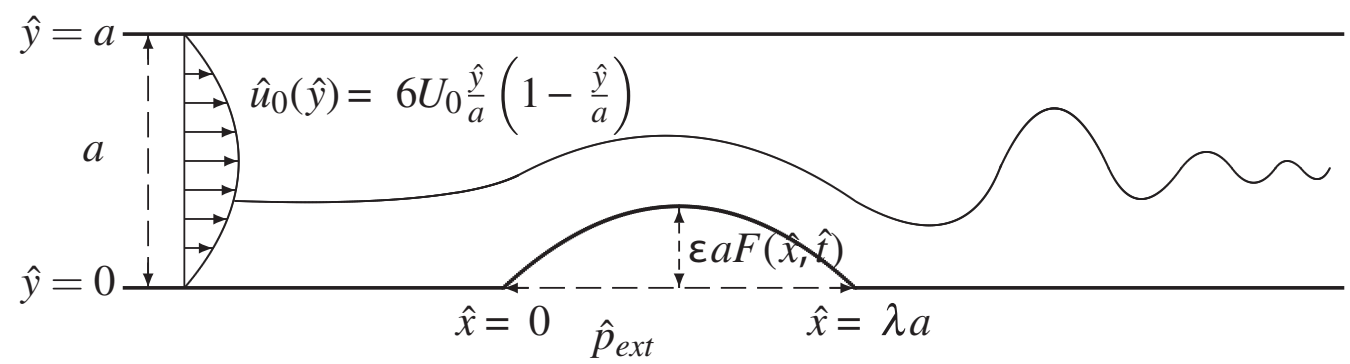

Figure 7. Diagram of the collapsible channel. All quantities are given in dimensional variables. The line in the middle of the channel indicates the streamline displacement due to the elastic wall deformation. From Pihler-Puzović \& Pedley (2014), figure 1.

channel, from one of the walls of which a section has been removed and replaced by a thin membrane under longitudinal tension $T$. This is much more susceptible to direct numerical simulation at moderate values of $R e$ (up to several hundred). Some results, by Luo \& Pedley (1996), are shown in figures 8 (steady) and 9 (unsteady). For these figures, the inlet Reynolds number was held fixed (at 300) and the dimensionless membrane tension $T$ was reduced (results for fixed $T$ and increasing $R e$ were similar but more difficult to obtain). Figure 8(a) shows how the membrane shape changes as $T$ is reduced: at high $T$ it is undeformed; as $T$ falls it becomes indented, though below a certain tension the indentation does not become more pronounced, it merely moves downstream, while a bulge develops upstream. The minimum channel width is plotted against $T^{-1}$ in figure 8(b), together with the corresponding curve from a 1D model (see below); circles show the value of $T$ at which bulging begins, and broken curves mark steady flow conditions which the time-dependent computations show to be unstable. The initial conditions for unsteady computations were taken to be the steady flow at a slightly lower $R e$. For
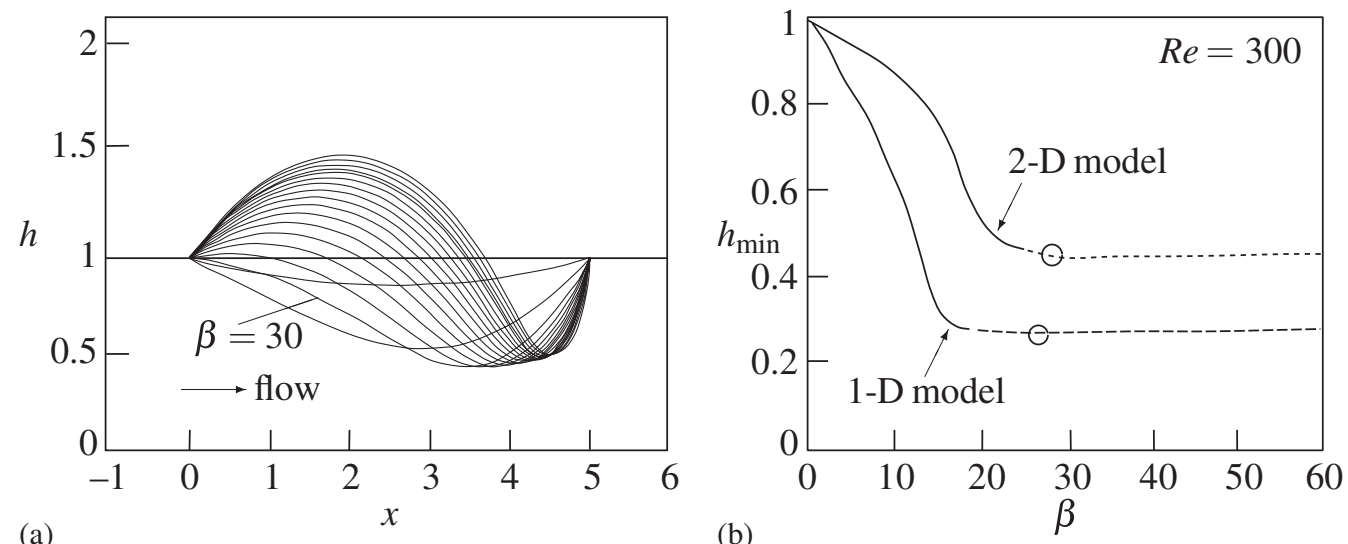

Figure 8. (a) Computed predictions of steady membrane shape at $R e=300$ and various values of tension parameter $\beta(\propto 1 / T)$. From Luo \& Pedley (1996). (b) Predictions of minimum channel width during steady flow, plotted against $\beta$ for fixed $\operatorname{Re}(=300)$. Bold solid and broken curves, from the 2D computations; fine solid and broken curves, from the 1D model. The broken curves represent steady states that are subsequently found to be unstable. Circles mark the value of $\beta$ at which upstream bulging first appears. From Pedley \& Luo (1998), figure 8. 

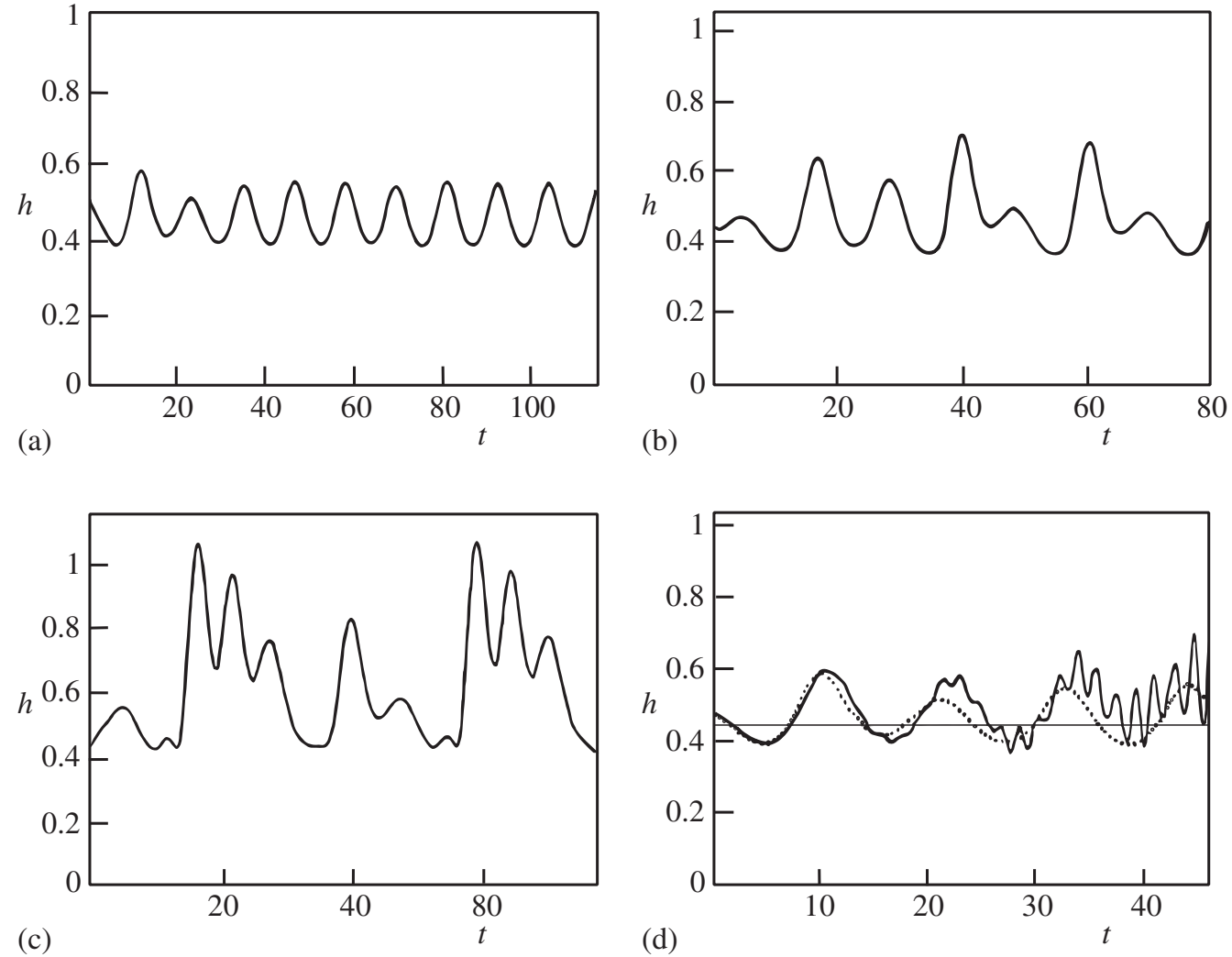

Figure 9. Membrane displacement $h$ at fixed $x=0.7$ as a function of time during self-excited oscillations. $R e=300$; (a) $\beta=30.0, M=0$, (b) $\beta=32.5, M=0$, (c) $\beta=35.0, M=0$, (d) $\beta=30.0, M=0.1$ (solid curve), $M=0$ (dotted curve). From Pedley \& Luo (1998), figures 9 and 10.

large $T$ the perturbation dies away. As $T$ is reduced below a critical value, the perturbation grows and an approximately sinusoidal oscillation develops (figure 9(a)), suggesting the presence of a supercritical Hopf bifurcation. At slightly smaller $T$, there is a period-doubling bifurcation (figure 9(b)), and at still smaller $T$ the behaviour becomes more complex still (figure 9(c)). When membrane mass is included, as well as tension, higher frequency flutter oscillations are superimposed on the oscillations, and indeed on the steady state when that is stable at zero mass (figure 9(d)); the flutter grows until it dominates and the computation breaks down. Much of the theoretical research in the field over the last 18 years has been directed at trying to understand the physics behind these results.

\section{Standard 1D model}

The standard 1D model was introduced, for steady flow, by Shapiro (1977). The governing equations for unsteady flow in a horizontal tube are as follows, with reference to figure 5:

$$
\text { conservation of mass } \frac{\partial A}{\partial t}+\frac{\partial(u A)}{\partial x}=0,
$$




$$
\begin{aligned}
& \text { conservation of momentum } \frac{\partial u}{\partial t}+u \frac{\partial u}{\partial x}=-\frac{1}{\rho} \frac{\partial p}{\partial x}-R(A, u, x) u \text {, } \\
& \text { elasticity (tubelaw) } \quad p-p_{e}=\tilde{P}(A, x),
\end{aligned}
$$

where $A(x, t)$ is the cross-sectional area, $u(x, t)$ is the fluid velocity averaged across the crosssection, $p(x, t)$ is the fluid pressure, $p_{e}$ is the external pressure (constant), $\rho$ is the fluid density and $\tilde{P}$ is the supposed relationship between the transmural pressure $\left(p-p_{e}\right)$ and $A$, possibly with an independent dependence on $x$ (see figure 1). The last term in (4) represents pressure drop due to viscosity, written as if for a linear dependence on $u$, but allowing $R$ to depend on $u$ and $x$ as well as $A$ : for Poiseuille flow in a circular tube, $R \propto A^{-2}$; in general $\partial R / \partial A<0$.

For steady flow with $\tilde{P}$ dependent only on $A$, the equations can be combined to give

$$
\frac{d A}{d x}=\frac{-R u A}{c^{2}-u^{2}}
$$

where

$$
c^{2}=\frac{A}{\rho} \frac{d \tilde{P}}{d A}
$$

$c$ is the speed of propagation of long, small-amplitude pressure waves along the tube when its cross-sectional area is $A$ (Pedley 1980). Note too that $u A$ is the constant flow rate. If, at some value of $x, u<c$ and $A>A_{0}$, then (6) shows that $A$ will decrease. Hence $c$ will decrease, because of the reducing slope of figure 1 , and $R$ will increase, with the result that $A$ will decrease more rapidly. If the tube is long enough, a point will be reached at which $u$ is predicted to equal $c$, and the model will break down. This is the condition of choking, which in practice can only be resolved by a change to upstream conditions. If area (or, equivalently, pressure) and flow rate are both prescribed upstream, choking will inevitably lead to unsteady conditions since there is no steady state.

For a finite length elastic segment, however, even if choking is not predicted, the above model cannot describe the increase in area required for the downstream end to be attached to a rigid tube of the same diameter as the upstream one. Therefore Cancelli \& Pedley (1985) suggested adding a term $-T A_{x x}$, where $T$ is a constant longitudinal tension, to the right-hand side of (5); this term would be correct in a $2 \mathrm{D}$ channel with small slope. They also noted that, at moderate or large Reynolds numbers, the flow will separate from all but the smallest indentations, causing additional energy loss. These authors assumed that such energy loss would exceed that arising directly from viscosity, and proposed that (4) be replaced by

$$
\frac{\partial u}{\partial t}+\chi u \frac{\partial u}{\partial x}=-\frac{1}{\rho} \frac{\partial p}{\partial x},
$$

where $\chi$ takes the value 1 upstream of the narrowest point in the vessel, $x_{s}$, (consistent with Bernoulli's theorem) and a constant value between 0 and 1 downstream of it, allowing for only incomplete pressure recovery. This modified 1D model, using an approximate but qualitatively realistic tube law, was then thoroughly investigated by Jensen \& Pedley (1989) and Jensen (1990, 1992).

The principal results for steady flow (Jensen \& Pedley 1989) were: (i) When there is no energy loss at the narrowest point, i.e. $\chi=1$ everywhere, then there is a critical value of the flow rate $Q=u A$ above which the steady problem has no solution - i.e. choking occurs. The critical value depends on the length and tension of the membrane, being higher for larger $T$. (ii) However, whenever there is some energy loss, i.e. $0<\chi<1$ for $x_{s}<x<1$, then a steady solution 


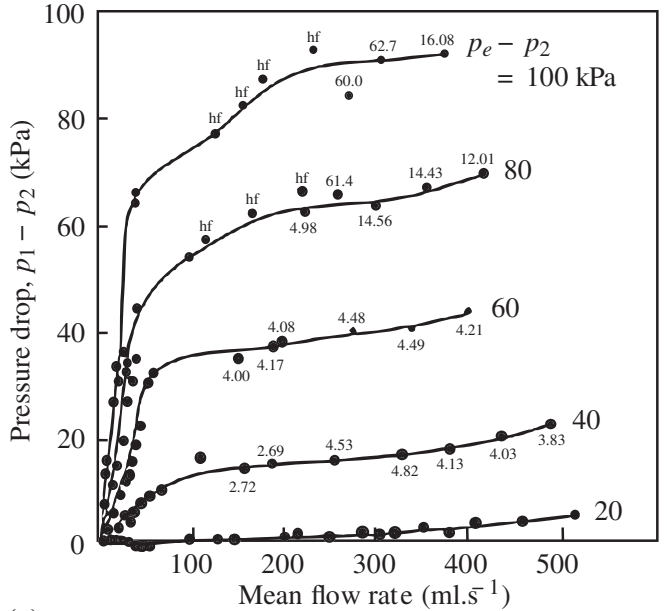

(a)

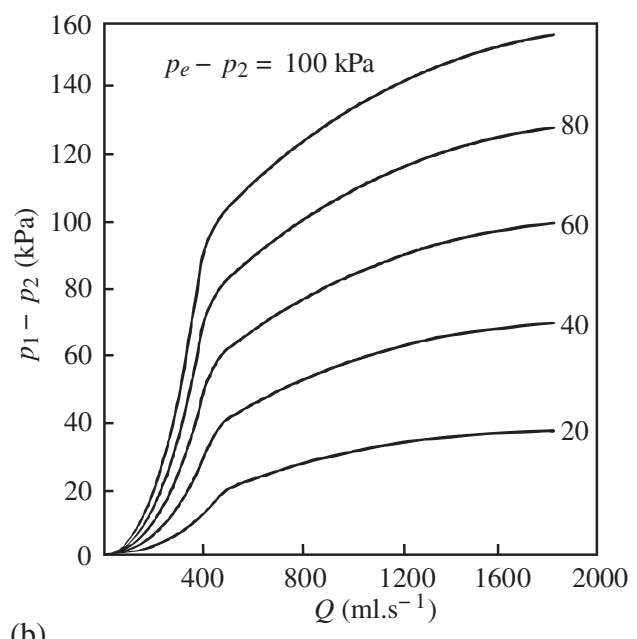

(b)

Figure 10. Comparison between (a) experimental measurements by Bertram (1986) of pressure drop $p_{1}-$ $p_{2}$ down the collapsible tube plotted against flow rate, for fixed values of $p_{e}-p_{2}$; and (b) curves calculated from the 1D model over a wider range of flow rates but otherwise using the same parameter values. The different symbols used in (a) to mark the measured points correspond to different downstream resistances used in the experiment, while the adjacent numbers refer to the frequency in $\mathrm{Hz}$ of observed oscillations , and for these cases time-averaged pressures are recorded. From Jensen \& Pedley (1989), figure 18.

exists for all positive values of flow rate and tension. Thus whether unsteady behaviour arises will depend on the stability of the steady solution, not its non-existence. A standard experimental measurement is of the overall pressure drop $p_{1}-p_{2}$ plotted against flow rate $Q$ at a fixed value of the transmural pressure difference at the downstream end of the collapsible segment, $p_{e}-p_{2}$. Results by Bertram (1986) are shown in figure 10(a), while the results from the 1D model are given in figure 10(b). They are qualitatively the same, except at low flow rates, when the absence of direct viscous resistance in the model is important (compare Eqs. (4) and (8)).

The numbers beside the experimental points in figure 10(a) are the frequencies of the selfexcited oscillations that arose in those experiments (the pressures plotted were mean values in those cases), as a result of instability. Linear and weakly nonlinear instability analyses for the

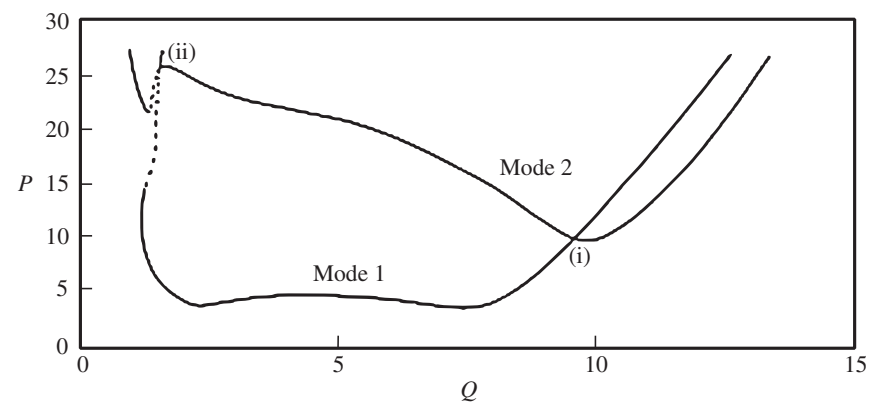

Figure 11. Stability boundaries for the first two modes of instability, plotted on the dimensionless $P-Q$ plane $\left(P \propto p_{e}-p_{2}\right)$, as predicted by the 1D model. The Hopf bifurcations are subcritical where the curves are dotted, supercritical elsewhere; (i) and (ii) are mode crossing points. From Jensen (1990), figure 8(a). 


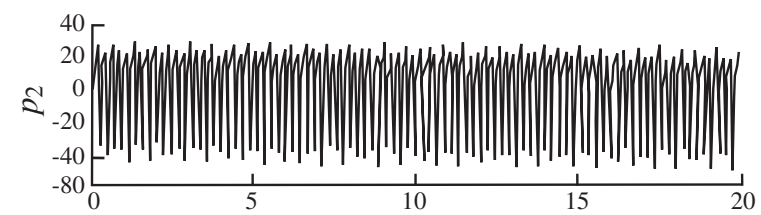

(a)

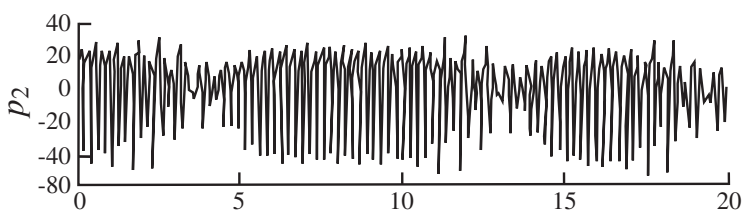

(b)
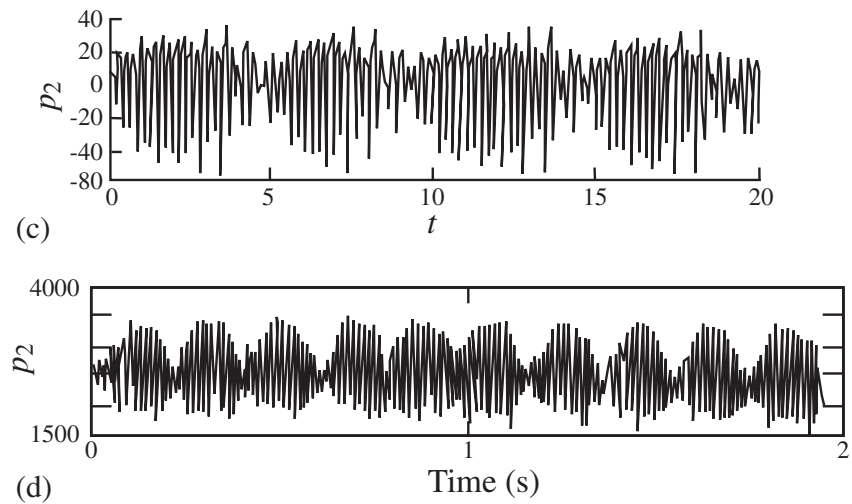

Figure 12. (a-c) Three time series during the development of 'bursting' oscillations: $P=30.25$ (a), 30.35 (b), 31.00 (c), from Jensen (1992). (d) An experimental time series for $p_{2}$ (arbitrary units) from Bertram et al (1991), figure 7.

1D model were performed by Jensen (1990), and he was able to calculate stability boundaries for different modes, plotted on the plane of dimensionless pressure drop $P$ and flow rate $Q$ in figure 11. Here Mode 1 refers to a wall displacement with one extremum, while Mode 2 has two extrema. Again, the prediction is qualitatively consistent with the experiments of Bertram (1986). For each mode the instability occurs through a Hopf bifurcation, which is supercritical almost everywhere, except where the plotted curves are dotted, near the mode crossing point (ii), where they are subcritical. Fully nonlinear computations from the 1D model for parameter values close to this point show interesting transitions between a high-frequency oscillation of almost constant amplitude to one which is severely modulated - see figure 12(a-c), which should be compared with the experimental figure 12(d). We should additionally note that the model was also used with parameter values appropriate to the computations of Luo \& Pedley (1996), and once more the agreement was pretty good (figure 8(b)). More discussion of the results of this 1D model is given in the original papers and in the review by Pedley \& Luo (1998).

\section{Rational 1D models}

The 1D model outlined above proved to be both simple to develop and remarkably successful in reproducing experimental and computational results. However, it contains a number of ad hoc 
assumptions, concerning viscous resistance, energy loss at flow separation, and longitudinal tension in the elastic segment of the tube or channel wall; these assumptions cannot be rationally justified by formal asymptotic analysis of the Navier-Stokes and elastic shell equations, and therefore are not strictly scientific. Here, therefore, we describe an approach to the 2D problem that is based on the interactive boundary-layer theory for high-Reynolds-number flow, first developed for tubes and channels with prescribed indentations by Smith (1976a, b). The 2D analysis that follows was initiated by Pedley \& Stephanoff (1985) but developed further by Guneratne \& Pedley (2006), Kudenatti et al (2012) and Pihler-Puzović \& Pedley (2013). The full 2D equations are derived and analysed elsewhere in this Symposium volume by Kudenatti et al (2015); here we merely give an outline and concentrate on the quasi-one dimensional, inviscid system that can be derived for wall displacements that are large compared with the boundary-layer thickness (Pedley \& Stephanoff 1985; Pihler-Puzović \& Pedley 2014).

We study high Reynolds number flow $(R e \gg 1)$ of incompressible viscous fluid, with mean velocity $U_{0}$, density $\rho$ and kinematic viscosity $\nu$, in a two-dimensional channel of width $a$ with a long slender indentation in the lower wall of a finite length $\lambda a, \lambda \gg 1$, and vertical displacement $\varepsilon a F(x, t), \varepsilon \ll 1$ (see figure 7). The flow rate at the entrance is taken to be fixed. Under the following non-dimensionalisation

$$
[\hat{x}, \hat{y}]=a[\lambda x, y], \quad[\hat{u}, \hat{v}]=U_{0}\left[u, \frac{v}{\lambda}\right], \quad \hat{p}=\rho U_{0}^{2} p, \quad \hat{t}=\frac{10 \lambda^{3} a}{U_{0}} t, \quad \operatorname{Re}=\frac{a U_{0}}{v},
$$

where indicates a dimensional variable, the flow field in the inviscid core, outside the boundary layers formed on the channel walls, is found to be:

$$
\begin{gathered}
u=u_{0}(y)+\varepsilon \tilde{A}(x, t) u_{0}^{\prime}(y)+\varepsilon^{2} u_{2}(x, y, t)+O\left(\varepsilon^{3}\right), \\
v=-\varepsilon \tilde{A}_{x}(x, t) u_{0}(y)+\varepsilon^{2} v_{2}(x, y, t)+O\left(\varepsilon^{3}\right), \\
p=-\frac{12 \lambda x}{\operatorname{Re}}+\varepsilon^{2}\left[P(x, t)+\tilde{A}_{x x}(x, t) \frac{1}{\lambda^{2} \varepsilon} \int_{0}^{y} u_{0}^{2}(\tilde{y}) d \tilde{y}\right]+O\left(\varepsilon^{3}\right),
\end{gathered}
$$

where $R e^{1 / 7} \leq \lambda \ll \operatorname{Re}$ and $R e^{-2 / 7} \ll \varepsilon \ll 1$ (see Pedley (2000)). Here $u_{0}(y)=6 y(1-y)$ is the Poiseuille velocity profile, $-12 \lambda / R e$ is the small pressure gradient required to drive the Poiseuille flow, $-\varepsilon \tilde{A}(x, t)$ is the lateral displacement of streamlines in the core flow, which would in the absence of the indentation stay straight, and $P$ is an unknown contribution to the pressure $p$. From (11) it can also be seen that

$$
p_{y}=\varepsilon^{2} \frac{5 \sigma}{6} \tilde{A}_{x x} u_{0}^{2}(y)+O\left(\varepsilon^{3}\right),
$$

where the parameter $\sigma=6 / 5 \lambda^{2} \varepsilon$ represents the importance or otherwise of the cross-stream pressure gradient.

We follow Pedley \& Stephanoff (1985) in assuming that the height of the indentation is much greater than the thickness of the boundary layers which form at the walls, but is still small compared to 1 , and $\sigma=O(1)$. This assumption allows us to proceed to $O\left(\varepsilon^{2}\right)$ in the $x$-momentum equation to obtain the following equation in the inviscid core

$$
u_{0} u_{2 x}+u_{0}^{\prime} v_{2}+\frac{\sigma}{12} \tilde{A}_{t} u_{0}^{\prime}+\tilde{A} \tilde{A}_{x}\left(\left(u_{0}^{\prime}\right)^{2}-u_{0} u_{0}^{\prime \prime}\right)=-P_{x}-\frac{5 \sigma}{6} \tilde{A}_{x x x} \int_{0}^{y} u_{0}^{2}(\tilde{y}) d \tilde{y} .
$$


Using the no-penetration boundary conditions,

$$
v=\varepsilon\left(u F_{x}+\frac{1}{10 \lambda^{2}} F_{t}\right) \quad \text { on } y=\varepsilon F(x, t) \quad \text { and } \quad v=0 \quad \text { on } y=1,
$$

we find the unknown velocity terms from (12) in the vicinity of $y=0$ and $y=1$. It follows that,

$$
\text { at } y=0: \quad 36\left(F \tilde{A}_{x}+\tilde{A} F_{x}+F F_{x}\right)+\frac{\sigma}{2} F_{t}+\frac{\sigma}{2} \tilde{A}_{t}+36 \tilde{A} \tilde{A}_{x}=-P_{x},
$$

and

$$
\text { at } y=1: \quad-\frac{\sigma}{2} \tilde{A}_{t}+36 \tilde{A} \tilde{A}_{x}=-P_{x}-\sigma \tilde{A}_{x x x} .
$$

On eliminating $P$ we obtain the following equation for the streamline displacement:

$$
\tilde{A}_{t}=\tilde{A}_{x x x}-\frac{1}{2} F_{t}-\frac{36}{\sigma}\left[F \tilde{A}+\frac{1}{2} F^{2}\right]_{x} .
$$

It can be seen that, upstream and downstream of the indentation, where $F \equiv 0,(15)$ reduces to the linearised Korteweg-de Vries equation, which supports downstream propagating waves (called vorticity waves because they owe their existence to the gradient of vorticity in the incoming Poiseuille flow) whose group velocity is three times their phase velocity. In their experiments, Pedley \& Stephanoff (1985) observed a train of such waves, with wave front speed approximately three times the wave crest speed, when the wall was forced with a given frequency. Moreover, by solving (15) for the appropriate $F(x, t)$, they confirmed quantitatively the applicability of the theory. Note that vorticity waves cannot be generated if the cross-stream pressure gradient, responsible for curvature of the core-flow streamlines, is negligible, as can be seen from (13) and (14) with $\sigma=0$.

In deriving Eq. (15) it is implicitly assumed that the boundary layers at the channel walls stay attached at all times and there is no breakaway separation. Numerical solution of the full 2D boundary-layer problem shows that small regions of reversed flow appear at both the upstream corner of the hump and at its downstream end (Guneratne \& Pedley 2006; Pihler-Puzović \& Pedley 2013) but, as argued by Pedley \& Stephanoff (1985), breakaway separation would occur at a later time and would not invalidate the above theory until the indentation of the wall had grown large. It should also be noted that, implicit in the interactive boundary-layer treatment, is the assumption that the incoming flow rate to the channel is prescribed and steady with, as stated above, large Reynolds number.

When the indentation is not prescribed but arises from deformation of an elastic membrane, (15) must be solved together with (14) and an equation that relates the deformation of the membrane to the pressure difference across it. For a long membrane under tension this equation, in dimensionless form, is

$$
M F_{t t}-T F_{x x}=P_{e x t}-P,
$$

where $T$ is the initial longitudinal tension, which is constant up to $O\left(\varepsilon^{2}\right), P_{\text {ext }}$ is the pressure external to the channel and $M$ represents the mass of the flexible wall. These quantities are related to the dimensional tension $\hat{T}$, external pressure $\hat{p}_{\text {ext }}$ and membrane mass per unit area $\hat{M}$ via

$$
\hat{T}=\varepsilon \rho U_{0}^{2} \lambda^{2} a T, \quad \hat{p}_{\text {ext }}=\varepsilon^{2} \rho U_{0}^{2} P_{\text {ext }} \quad \text { and } \quad \hat{M}=10^{2} \varepsilon \rho \lambda^{6} a M .
$$

Equation (16), for the case of non-zero membrane mass, replaces (5). 
In earlier studies in which the full 2D boundary-layer problem was solved, as in the NavierStokes computations of Luo \& Pedley (1996), membrane inertia was ignored $(M=0)$. Guneratne \& Pedley (2006) solved the steady flow problem for cases in which the transmural pressure was prescribed to be zero at the upstream end of the system, $x \rightarrow-\infty$ (and hence at $x=0$ when $\sigma=0$ ). In their computations they fixed $P_{\text {ext }}$ and $\sigma$ and considered a range of values of $T$. At large $T$ the membrane remained almost undeformed whatever the value of $P_{\text {ext }}$. As $T$ was reduced, deformation increased, and multiple solution branches were revealed, corresponding to deformations with different numbers of extrema (different mode numbers), as shown for $P_{\text {ext }}=1$ and $\sigma=0$ in figure 13(b). We may note that there are ranges of $T$ for which no solution exists; the saddle-node bifurcation at $T \approx 1$ can be interpreted as evidence of choking when flow rate and pressure are prescribed upstream. When $P_{\text {ext }}=0$ a solution always exists in which the membrane is undeformed, but other solutions bifurcate from it at particular values of $T$ (see the case of $P_{\text {ext }}=0.01 \approx 0$ in figure 13(a)); these values can be calculated independently as eigenvalues of the linearised problem for small deformations. Non-zero values of $\sigma$ make no qualitative difference to these findings. Pihler-Puzović \& Pedley (2013) repeated the above steady-flow computations but with zero transmural pressure prescribed downstream, at $x=1$ or further downstream. They found that a solution exists for all values of $T, P_{\text {ext }}$ and $\sigma$ that were tested, but it was non-unique when the position downstream at which pressure was prescribed was at $x>1.2$.

In order to investigate the possible development of self-excited oscillations, Pihler-Puzović \& Pedley (2013) computed the stability characteristics of the steady solutions by numerically solving the unsteady membrane-plus-boundary-layer equations, still in the absence of membrane inertia. They found that perturbations to the steady solution blow up exponentially fast when the pressure is prescribed upstream, even for large values of $T$, because the problem is mathematically ill-posed (see also Kudenatti et al (2012)). However, when the pressure is fixed downstream, at $x \leq 1.2$, the steady solution is completely stable; some eigenmodes, calculated from linearised equations, consist of decaying oscillations, but the most slowly decaying mode is always non-oscillatory. When pressure is prescribed further downstream, so that the steady solution is non-unique, the 'natural', partially collapsed steady solution does become unstable, but always without oscillation; the other, outwardly bulging, solution is stable in those cases.

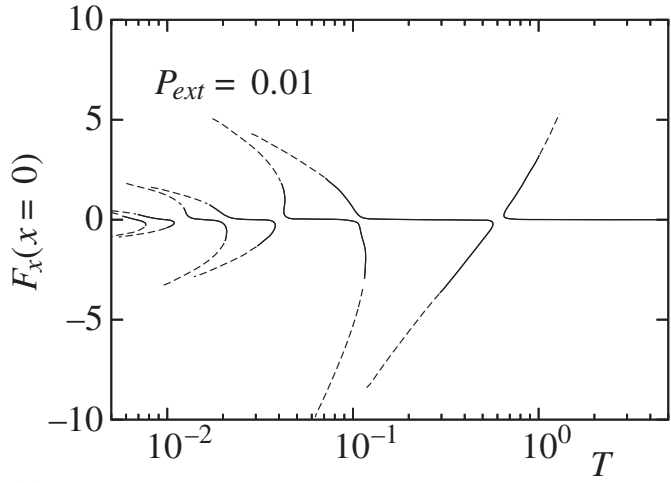

(a)

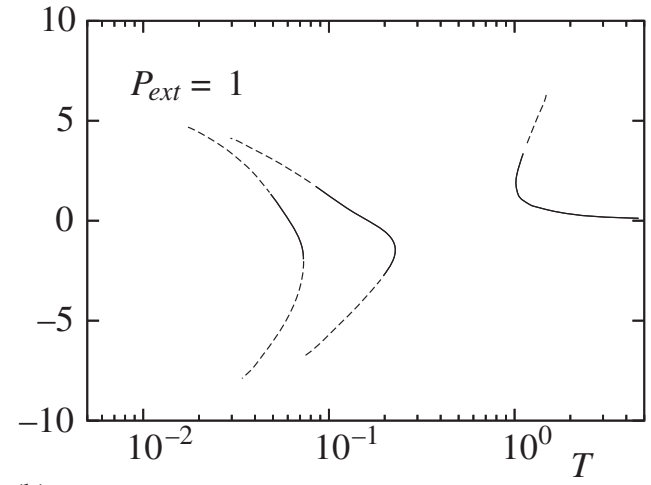

(b)

Figure 13. Bifurcation diagrams with $\sigma=0$ for (a) $P_{\text {ext }}=0.01$ and (b) $P_{\text {ext }}=1$ : the membrane slope at its upstream end is plotted against the non-dimensional tension. The dashed curves indicate solutions in which there are regions of flow reversal. From Guneratne \& Pedley (2006), figure 6. 
Surprisingly, the results do not change qualitatively for non-zero values of $\sigma$ : the possibility of vorticity waves does not lead to oscillatory instability.

The effect of non-zero membrane inertia on the above results was investigated by PihlerPuzović \& Pedley (2014) using the 1D model given by Eqs. (14)-(16). Implicitly, therefore, the steady flow rate, not the pressure, is prescribed far upstream. In order to avoid the reflection of propagating disturbances from the ends of the system, only finitely far upstream and downstream, appropriate absorbing boundary conditions were carefully applied. The first finding was that a steady solution exists whenever $T$ exceeds a (fairly small) critical value, However, unlike the full 2D boundary-layer computations, this model leads to only mode- 1 membrane deformations. Moreover, for $P_{\text {ext }}=0$, the only steady solution is that of zero deformation unperturbed Poiseuille flow in a parallel-sided channel - so the multiple solution structure found by Guneratne \& Pedley (2006) arises only in the full boundary-layer problem.

Unsteady solutions were obtained from either a smooth increase of external pressure $P_{\text {ext }}$ from zero, or a spike in $P_{\text {ext }}$. When $M=0$, the unsteady solution invariably tends to the steady one at large times, though transient vorticity waves are generated downstream when the cross-stream pressure-gradient parameter $\sigma$ is non-zero. On the other hand, when both $M$ and $\sigma$ are non-zero a flutter instability always arises at large time (figure 14(a-b)). Very similar results were obtained from the full Navier-Stokes computations at moderate Reynolds number, as performed by Luo \& Pedley (1998). The initial deformation is of mode 1, as for the corresponding steady solution, but the oscillations that develop at large times are generally of higher mode number; for instance, the example of figure 14(b) is a mode- 2 oscillation. Figure 15 shows the results of a linear instability calculation, indicating how the most unstable mode number depends on $M, P_{\text {ext }}$ and $\sigma$. Even for small values of $M$, the fluid loading on the membrane has little effect on the frequencies of the computed oscillations of the stretched membrane, which are close to $n \pi \sqrt{T / M}$ for integer $n$ (e.g. $n=1$ for the initial decaying oscillations in figure 14(b) and $n=2$ for the large time unstable flutter). However, as stated above, the oscillations do not grow unless $\sigma \neq 0$, indicating the importance of streamline curvature, with the corresponding vorticity-wave generation, for the instability. We may note that, according to this model, the oscillations never saturate to give constant, but finite, amplitude oscillations. The amplitude continues to grow until the computation breaks down, when a second, unphysical branch of steady solutions is encountered; these solutions are exponentially unstable. Thus, neither the full, interactive boundary-layer model,

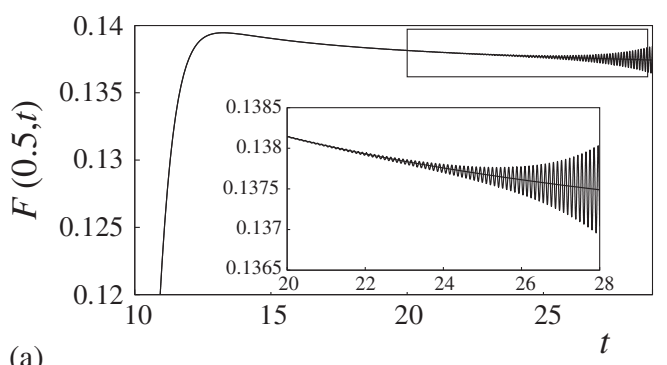

(a)

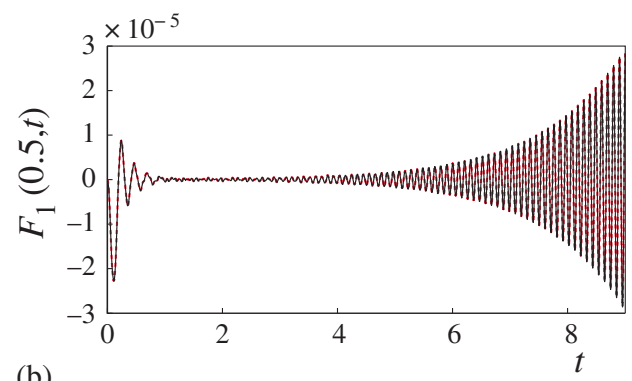

(b)

Figure 14. (a) Time evolution of membrane displacement $F(0.5, t)$ at $x=0.5 ; \sigma=T=P_{\text {ext }}=$ $1, M=0.01$ (curve with oscillations) and $M=0$ (curve without oscillations); initially $P_{\text {ext }}$ is increased smoothly. (b) Time evolution of $F_{1}(0.5, t)$, the difference between the $M=0.01$ and $M=0$ cases; $\sigma=T=P_{\text {ext }}=1$; initially the system was perturbed abruptly. From Pihler-Puzović \& Pedley (2014), figures 5(a) and 6(a). 


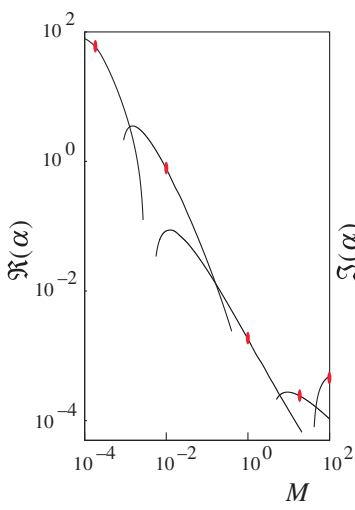

(a)

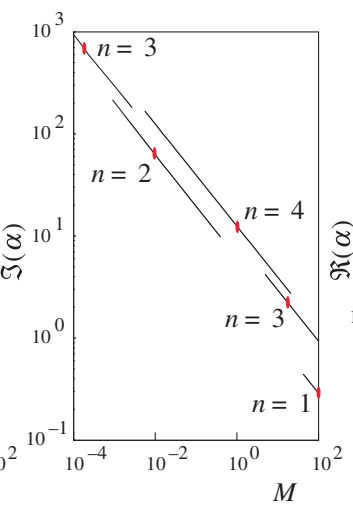

(b)

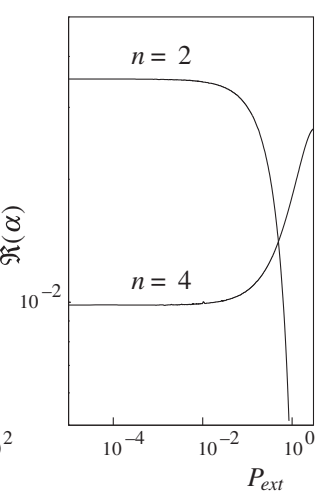

(c)

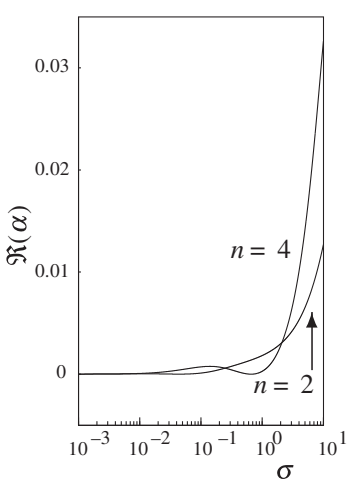

(d)

Figure 15. Dependence of (a) the growth rate $\Re(\alpha)$ and (b) the frequency $\Im(\alpha)$ of the fastest growing eigenvalue on the membrane mass $M$ when $P_{\text {ext }}=T=\sigma=1$. Vertical marks are drawn for clarity to relate real and imaginary parts of selected eigenvalues. The change in $\Re(\alpha)$ with (c) the external pressure $P_{\text {ext }}$ and (d) cross-stream pressure gradient parameter $\sigma$ for selected eigenvalues when (c) $\sigma=T=M=1$ and (d) $P_{\text {ext }}=T=M=1$. In (b-d), $n$ is the number of extrema in the elastic wall deformation of the corresponding eigenvector. From Pihler-Puzović \& Pedley (2014), figure 8.

nor the 1D version of it given by (14) - (16), is capable of explaining the large-amplitude selfexcited oscillations that are observed in experiments on tubes (figure 6) and 2D Navier-Stokes computations (figure 9), especially for zero membrane inertia.

The main difference between the traditional, ad hoc, 1D model of Eqs. (3)-(5) and the essentially inviscid model leading to (13) and (14) is the appearance in the momentum equation (4) of the term $R u$ representing viscous resistance. A rational way of including viscosity in a 1D model has been developed by Jensen \& Heil (2003), Stewart et al $(2009,2010)$ and Xu et al (2013, 2014). In this model the undisturbed state is taken to be parallel-sided $(F=0)$ through the device of supposing the external pressure to vary linearly with $x$ in the same way as for Poiseuille flow in the channel. Moreover, the lengths of the membrane and the up- and downstream rigid segments, and the length scale of all deformations, are taken to be sufficiently large that quasisteady Poiseuille flow can be assumed at every location in the channel. That is, $\lambda \gg R e \geq O(1)$ and, everywhere,

$$
u=6 q \frac{(1-y)(y-F)}{(1-F)^{3}},
$$

where $q(x, t)=\int_{F}^{1} u d y$ is the flow rate. Note that the Reynolds number is not assumed to be necessarily very large. The longitudinal momentum equation is integrated across the channel, and the governing equations reduce to the following, where we now follow the model's authors (Jensen \& Heil (2003) etc.) in writing $h \equiv A \equiv 1-F$ :

$$
\begin{gathered}
\frac{\partial h}{\partial t}+\frac{\partial q}{\partial x}=0 \\
\frac{\partial q}{\partial t}+\frac{6}{5} \frac{\partial\left(q^{2} / h\right)}{\partial x}=-h \frac{\partial p}{\partial x}-12 \frac{R q}{h^{2}} \\
p=p_{e}(x)-T \frac{\partial^{2} h}{\partial x^{2}}
\end{gathered}
$$




$$
p_{e}(x)=12\left(L_{2}+1-x\right) R .
$$

Here $R$ in (19) and (21) is a constant resistance parameter (inversely proportional to the Reynolds number), and the convective inertia term has a factor 6/5 instead of 1 (as in (4)) because of the parabolic velocity profile (17). Xu et al (2013) showed that this difference is qualitatively unimportant. $L_{2}$ in (21) is the dimensionless length of the downstream rigid part of the channel, with pressure specified to be zero at the downstream end. Eliminating $p$ from (19) and (20) using (21) gives

$$
\frac{\partial q}{\partial t}+\frac{6}{5} \frac{\partial\left(q^{2} / h\right)}{\partial x}=T h \frac{\partial^{3} h}{\partial x^{3}}+12 R\left(h-\frac{q}{h^{2}}\right) ;
$$

this equation together with (18) must be solved subject to boundary conditions

$$
h(0, t)=1, h(1, t)=1, q(0, t)=1,
$$

and

$$
T \frac{\partial^{2} h}{\partial x^{2}}=-\left[12 R(q-1)+\frac{\partial q}{\partial t}\right] L_{2} \quad \text { at } x=1 .
$$

The last of these incorporates the resistive and inertial pressure drop along the downstream rigid channel. If the pressure, not the flow rate, were prescribed at the upstream end of the system $\left(x=-L_{1}\right.$, say), then $q(0, t)=1$ would be replaced by another condition like (24).

More than one type of sustained oscillation has been found with this model, using both numerical simulations and sophisticated asymptotic analysis. With a 2D model, Jensen \& Heil (2003), extended by Stewart et al (2010), discovered mode-1 sloshing oscillations at high tension, in which the in-and-out motion of the membrane forces significant oscillatory volume flow in the rigid parts of the channel; energy can be transferred from the inflow to the oscillations if the downstream rigid segment is longer than the upstream one. Stewart et al (2009) showed how these results can be obtained from the 1D model. This type of oscillation, however, requires the upstream pressure to be fixed, not the flow rate. Progress with fixed inflow rate has been made by Xu et al $(2013,2014)$. In the first of these papers the system is examined for cases in which the downstream rigid segment is of shorter or comparable length to the membrane, while the second paper considers a much longer downstream segment $\left(L_{2} \gg 1\right)$. In each case the authors perform a linear stability analysis of the undisturbed state $(h \equiv 1)$, using both numerical and asymptotic methods to solve the resulting eigenvalue problem. Even this apparently simple problem reveals how intricate the system is, because as stability boundaries are drawn in the $R-T$ parameter space, transcritical (divergent) and Hopf (oscillatory) bifurcations occur, sometimes simultaneously. Yet more intricate weakly nonlinear analyses show in particular a wealth of different unsteady behaviours that can arise in the neighbourhood of a particular organising point ( $R \rightarrow 0, T=T_{20} \approx 0.0304$ ). The analysis is supplemented by numerical solution of (18) and (22). The results indicate two possible states of sustained oscillations that can arise. (i) For $L_{2}=O(1)$ an oscillatory mode- 2 instability grows until the membrane collapses quite violently into near contact with the opposite wall of the channel (so-called slamming), generating waves that propagate and dissipate the energy of the collapse, which therefore relaxes before starting again. (ii) For $L_{2} \gg 1$, the stability boundary of the Hopf bifurcation in the $R-T$ plane, for $T-T_{20}$ small and negative and $R$ not very small, has three distinct branches. From the upper one of these, with $T_{20}-T=O\left(L_{2}^{-1}\right)$ and $R=O\left(L_{2}^{-1 / 2}\right)$, finite-amplitude sustained oscillations can arise, without the near collapse associated with 'slamming'. The oscillations result from a resonance between mode- 2 and mode- 1 unstable oscillations, a resonance that is prevented from 
growing too large by viscous dissipation in the downstream rigid segment. A crucial confirmation of the validity of these $1 \mathrm{D}$ analytical results comes from direct numerical simulation of the 2D system, using the full Navier-Stokes equations; this was also performed by Xu et al (2014), for particular parameter values in the correct range. Sustained oscillations of the correct form did indeed develop. It is of interest to note that the amplitude was sufficiently small that there was no evidence of vorticity waves in the flow downstream of the membrane.

We can sum up by saying that, although full computations of the 2D problem predicted selfexcited oscillations nearly twenty years ago (Luo \& Pedley 1996), the work of Xu et al (2014) provides the first rational analysis of such oscillations in the absence of membrane inertia. Interestingly, flow separation and vorticity waves do not arise and are therefore not necessary for self-excited oscillations to be generated. Of course, there is a big difference between the Xu et al (2014) model and that of Luo \& Pedley (1996), because the basic state for the former was that of no deformation, whereas in the latter case it involves large deformation and flow separation. Therefore, it cannot yet be said that "the collapsible tube problem" is fully solved, even in two dimensions.

\section{References}

Bertram C D 1986 Unstable equilibrium behaviour in collapsible tubes. J. Biomech. 19: 61-69

Bertram C D, Raymond C J and Pedley T J 1991 Application of nonlinear dynamics concepts to the analysis of self-excited oscillations of a collapsible tube conveying a flow. J. Fluids Struct. 5: 391-426

Brook B S, Falle S A E G and Pedley T J 1999 Numerical solutions for unsteady gravity-driven flows in collapsible tubes: evolution and roll-wave instability of a steady state. J. Fluid Mech. 396: 223-256

Cancelli C and Pedley T J 1985 A separated flow model for collapsible tube oscillations. J. Fluid Mech. 157: 375-404

Gavriely N, Shee T R, Cugall D W and Grotberg J B 1989 Flutter in flow-limited collapsible tubes: A mechanism for generation of wheezes. J. Appl. Physiol. 66: 2251-2261

Guneratne J and Pedley T J 2006 High-Reynolds-number steady flow in a collapsible channel. J. Fluid Mech. 569: 151-184

Hargens A R, Millard R W, Pettersson K and Johansen K 1987 Gravitational haemodynamics and oedema prevention in the giraffe. Nature 329: 59-60

Hicks J W and Badeer H S 1989 Siphon mechanism in collapsible tubes: application to circulation in the giraffe head. Am. J. Physiol. 256: R567-R571

Hyatt R E, Schilder D P and Fry D L 1958 Relationship between maximum expiratory flow and degree of lung inflation. J. Appl. Physiol. 13: 331-336

Jensen O E 1990 Instabilities of flow in a collapsed tube. J. Fluid Mech. 220: 623-659

Jensen O E 1992 Chaotic oscillations in a simple collapsible-tube model. ASME J. Biomech. Eng. 144: 5559

Jensen O E and Heil M 2003 High-frequency self-excited oscillations in a collapsible-channel flow. $J$. Fluid Mech. 481: 235-268

Jensen O E and Pedley T J 1989 The existence of steady flow in a collapsed tube. J. Fluid Mech. 206: 339374

Kudenatti R B, Bujurke N M and Pedley T J 2012 Stability of two-dimensional collapsible-channel flow at high Reynolds number. J. Fluid Mech. 705: 371-386

Kudenatti R B, Bujurke N M and Pedley T J 2015 Chapter in this volume

Luo X Y and Pedley T J 1996 A numerical simulation of unsteady flow in a 2-D collapsible channel. $J$. Fluid Mech. 314: 191-225

Luo X Y and Pedley T J 1998 The effects of wall inertia on flow in a 2-D collapsible channel. J. Fluid Mech. 363: 253-280

Pedley T J 1980 The fluid mechanics of large blood vessels. Cambridge University Press 
Pedley T J 2000 Blood flow in arteries and veins. In: Batchelor G K, Moffatt HK, Worster MG (eds) Perspectives in fluid dynamics. Cambridge University Press, pp 105-158

Pedley T J, Brook B S and Seymour R S 1996 Blood pressure and flow rate in the giraffe jugular vein. Phil. Trans. R Soc Lond. B 351: 855-866

Pedley T J and Luo X Y 1998 Models of flow and self-excited oscillations in collapsible tubes. Theor. Comp. Fluid Dyn. 10: 277-294

Pedley T J and Stephanoff K D 1985 Flow along a channel with a time-dependent indentation in one wall: The generation of vorticity waves. J. Fluid Mech. 160: 337-367

Pihler-Puzović D and Pedley T J 2013 Stability of high-Reynolds-number flow in a collapsible channel. $J$. Fluid Mech. 714: 536-561

Pihler-Puzović D and Pedley T J 2014 Flutter in a quasi-one-dimensional model of a collapsible channel. Proc. R. Soc. Lond. A 470: 20140,015

Shapiro A H 1977 Steady flow in collapsible tubes. ASME J. Biomech. Eng. 99: 126-147

Smith F T 1976a Flow through constricted or dilated pipes and channels: Part i. Q. J. Mech. Appl. Math. 29: $343-364$

Smith F T 1976b Flow through constricted or dilated pipes and channels: Part ii. Q. J. Mech. Appl. Math. 29: $365-376$

Stewart P S, Heil M, Waters S L and Jensen O E 2010 Sloshing and slamming oscillations in collapsiblechannel flow. J. Fluid Mech. 662: 288-319

Stewart P S, Waters S L and Jensen O E 2009 Local and global instabilities of flow in a flexible-walled channel. Eur. J. Mech. B/Fluids 28: 541-557

Xu F, Billingham J and Jensen O E 2013 Divergence-driven oscillations in a flexible-channel flow with fixed upstream flux. J. Fluid Mech. 723: 706-733

Xu F, Billingham J and Jensen O E 2014 Resonance-driven oscillations in a flexible-channel flow with fixed upstream flux and a long downstream rigid segment. J. Fluid Mech. 746: 368-404 